\title{
Inhomogeneous Structure Formation May Alleviate Need for Accelerating Universe
}

\author{
Johan Hansson* and Jesper Lindkvist \\ Department of Physics, Luleå University of Technology, SE-971 87 Luleå, Sweden
}

\begin{abstract}
When taking the real, inhomogeneous and anisotropic matter distribution in the semi-local universe into account, there may be no need to postulate an accelerating expansion of the universe despite recent type Ia supernova data. Local curvatures must be integrated (over all space) to obtain the global curvature of the universe, which seems to be very close to zero from cosmic microwave background data. As gravitational structure formation creates bound regions of positive curvature, the regions in between become negatively curved in order to comply with a vanishing global curvature. The actual dynamics of the universe is altered due to the self-induced inhomogeneities, again more prominently so as structure formation progresses. Furthermore, this negative curvature will increase as a function of time as structure formation proceeds, which mimics the effect of "dark energy" with negative pressure. Hence, the "acceleration" may be merely a mirage. We make a qualitative and semi-quantitative analysis, for pedagogical reasons using newtonian gravity corrected for special relativistic effects (which works surprisingly well) to corroborate and illustrate/visualize these statements. This article may be seen as an attempt to communicate to a larger number of people the necessity of starting to take seriously the real, observed inhomogeneous distribution and the nonlinearities of nonperturbative general relativity, and their impact on the dynamics and behavior of the cosmos instead of allowing an oversimplified cosmological model to generate a consensus world-view of a cosmos allegedly dominated by mysterious dark energy.
\end{abstract}

Keywords: Inhomogeneous Cosmology, Dark Energy, Structure Formation, Non-Accelerating Universe.

Measurements since the late 1990s on type Ia supernovae $(\mathrm{SN})[1,2]$ surprisingly seemed to indicate that the universe accelerates its expansion at the present epoch, instead of the deceleration expected if gravity is universally attractive. This finding has resulted in a "neo-standard" interpretation where the present universe is believed to be dominated by some exotic "dark energy" with negative pressure. However, when taking the observed inhomogeneous structure of the universe into account, and considering the real (geodesic) paths of observed SN light, such a hypothesis might be superfluous.

i) If we assume that luminous matter (and by necessity gas, dust and plasma for star formation) is a good "tracer" of regions of higher than average density, almost all photons from distant objects that reach us on earth must have traversed regions with little or no matter with which it can interact electromagnetically. The light from distant objects "zigzag" through the maze defined by gravitationally bound objects (galaxies, galaxy clusters, superclusters) making the real (geodesic) path longer than the one calculated from standard, perfectly isotropic and homogeneous, FRWcosmology, the more so the longer structure formation has progressed $(z \leq \sim 1)$ [3].

ii) The actual dynamics of the universe is altered due to the self-induced inhomogeneities, again more prominently so as structure formation progress.

We will, deliberately from a pedagogical standpoint, employ a heuristic model using newtonian gravity, with

*Address correspondence to this author at the Department of Physics, Luleå University of Technology, SE-971 87 Luleå, Sweden; Tel: +46 (0)920 491072; Fax: +46 (0)920 491074; E-mail: c.johan.hansson@ltu.se

1874-3811/10 special relativistic corrections, both for the ease of visualization/interpretation and the much simpler (linear) mathematics compared to full-blown (nonlinear) general relativity, in which not even the two-body case is analytically solvable ${ }^{1}$. Even though newtonian gravity is not completely mathematically consistent in an infinite universe, due to its dependence on boundary conditions infinitely far away, we can side-step this by treating the Big Bang as an "explosion" in a pre-existing (newtonian) space. As can be seen in Fig. (2), this special relativistically corrected $\left(p=m_{0} v \rightarrow p=m_{0} v / \sqrt{1-v^{2} / c^{2}}\right)$ newtonian model comes surprisingly close to the general relativistic FRW-model, especially for low $z$ as expected.

In general relativity, gravitationally bound systems have a positive spacetime curvature. At the same time we know, from observations of the cosmological microwave background radiation (CMBR) [6-8], that the global geometry of the universe most probably is flat. This means that the curvature between gravitationally bound systems (solar systems, galaxies, galaxy clusters, etc) must be negative. This conclusion applies to all globally flat universes with (semi-)localized gravitationally bound systems.

For a truly exact description, we would need to know the energy-momentum tensor $\left(T_{\mu v}\right)$ at each point between us and the distant $\mathrm{SN}$, which is physically impossible. And even if we had such perfect information, it would still be

${ }^{1}$ General relativistic treatments, with specific simplifying assumptions, are covered in [4],[5]

2010 Bentham Open 
mathematically inconceivable to solve the resulting Einstein equations,

$$
R_{\mu v}-\frac{1}{2} g_{\mu v} R=\kappa T_{\mu v}
$$

to deduce the local curvature at each point in terms of the Riemann curvature tensor, due to the complexity of the equations - ten coupled, nonlinear PDEs. (The real lure of assuming perfect homogeneity and isotropy is that Eq.(1) then simplifies to two coupled linear ODEs, Friedmann's eqs., in which the total dynamical behavior of such FRWmodel universes is contained solely in $a(t)$, the cosmic scale factor.)

One hope would be that an approximate model could be used to determine an "effective" curvature for bound systems and for the space in-between. It would define a semi-local mean curvature parameter, $k$, for the regions bound/between. (This parameter is related to the scalar curvature $R=R_{\mu}^{\mu}$, averaged over the region, $\langle R\rangle$.) Even in such a "coarse-grained" inhomogeneous model, the cosmic scale factor $a(t)$ will be scale-dependent, as noted already by de Vaucouleurs ${ }^{2}$ [9].

In terms of $\Omega=\rho / \rho_{\text {crit }}$ (where $\rho_{\text {crit }}$ is the density required for flatness):

$\Omega_{\text {global }}=1$,

$\Omega_{\text {bound }}>1$,

$\Omega_{\text {between }}<1$.

Or, stated in terms of the mass-energy density: $\rho_{\text {global }}=\rho_{\text {crit }}, \rho_{\text {bound }}>\rho_{\text {crit }}, \rho_{\text {between }}<<\rho_{\text {crit }}$. (However, one should keep in mind that the very definition and usage of $\Omega$ assumes homogeneity and isotropy.)

As matter preferentially clumps in well localized objects (stars, etc), the majority of the photons that reach us travel mainly in "under-dense" (negatively curved) $\Omega<1$ space. By observing light we are thus automatically biased to measure an "apparent" curvature which is less than the actual global curvature. ${ }^{3}$ Light from a SN source will, in a universe which exhibits gravitational clumping/structure formation, always be switched towards a seemingly more negatively curved universe. For a universe with zero global curvature, the SN light will thus approach the curve for an open universe, see Fig. (1).

Another compelling property is that this negative curvature effect will automatically mimic a very small

\footnotetext{
${ }^{2}$ "This leads one to view the Hubble parameter as a stochastic variable, subject in the hierarchical scheme to effects of local density fluctuations on all scales."

${ }^{3}$ Neutrinos and gravitational waves should show less bias as they can travel unhindered through huge amounts of matter without interacting appreciably. This means that both negative and positive curvature effects contribute, which reduces the bias.
}

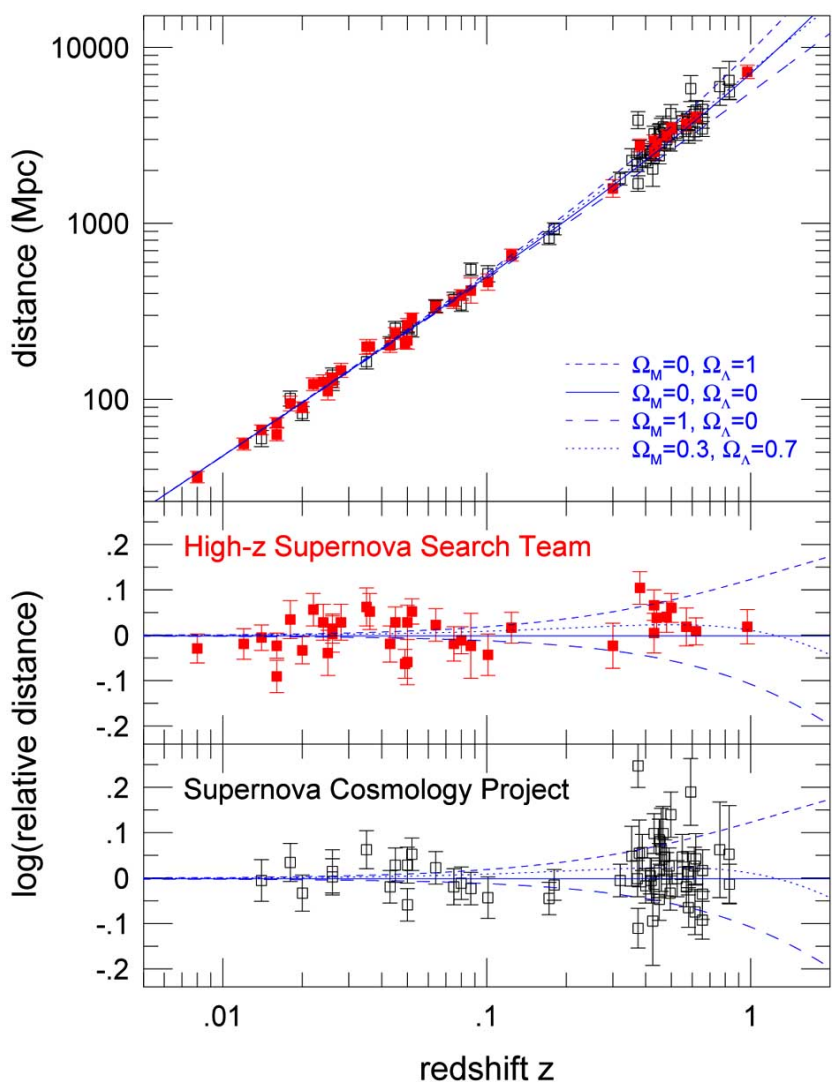

Fig. (1). Shown [10] are the original data points of the High-Z Supernova team (filled squares) [1], and the Supernova Cosmology Project (open squares) [2]. The dashed line is the theoretical prediction for a homogeneous and isotropic (FRW) universe which is flat and without cosmological constant $\left(\Omega_{M}=1, \Omega_{\Lambda}=0\right)$. The solid line is the corresponding prediction for an empty (open) universe ( $\Omega_{M}=0, \Omega_{\Lambda}=0$ ). Also shown (short dashes) is the theoretical prediction for a flat universe with solely a dark energy component ( $\Omega_{M}=0, \Omega_{\Lambda}=1$ ). The dotted line is the solution currently favored for the SN Ia data by both experimental groups ( $\Omega_{M}=0.3, \Omega_{\Lambda}=0.7$ ). For $z>1$, where unfortunately also observational measurements become increasingly difficult, it starts to deviate towards the $\left(\Omega_{M}=1, \Omega_{\Lambda}=0\right)$ line. The "neostandard" explanation for this is that $\Omega_{\Lambda}$ has become dominant only fairly recently $(z \leq 1)$. Observed $\mathrm{SN}$ photons within a globally flat universe will always tend towards the line for the open universe, due to inhomogeneous structure formation.

cosmological constant, beginning to "dominate" at an epoch when a significant amount of structure has evolved. Before structure formation through gravitational condensation becomes effective $(1100>>>4)$, all space will have roughly the same curvature $(k \sim 0)$. However, structure formation will produce bound systems with increasing $\Omega_{\text {bound }}$, which means that $\Omega_{\text {between }}$ will be a decreasing function of time. Hence, the space in-between bound systems will asymptotically approach $\Omega=0$ with time (underdensity regions being diluted by expansion), simulating an accelerated expansion. 
After these general considerations, we now turn to the newtonian model to qualitatively and semi-quantitatively, make our case about ii), the dynamics. First, it is possible to deduce analytical results from a maximally inhomogeneous and anisotropic distribution - a two-body problem.

We first define the "homogeneous acceleration", denoted $a_{h}$. This is the acceleration that would be experienced by an object for a perfectly homogeneous and isotropic distribution on all scales. Simply put, if a test particle is somewhere in a homogeneous sphere, the only net effect of gravitation is the mass within a smaller sphere with a radius equal to the distance, $d$, from the test mass to the center. The acceleration for this test mass becomes

$a_{h}=-\frac{G m}{d^{2}}$,

where $m$ is the mass of the small sphere. This mass can be expressed by the volume ratio times the total mass of the distibution, $M$,

$m=M \frac{d^{3}}{R^{3}}$,

where $R$ is the radius of the total volume. The acceleration can thus be expressed as

$a_{h}=-\frac{G M d}{R^{3}}$.

If we know the total mass, then one way of deciding $R$ is to check the mean-distance of the observed supernovas. If we assume that we can see all supernovas in the observable universe, we can then just take the mean-value of $d$ and multiply by four-thirds to get the radius, as the geometric center lies at three-fourths of the radius in a cone-fragment of a homogeneous sphere. The expresion for the acceleration becomes

$a_{h}=-\frac{27 G M d}{64\langle d\rangle^{3}}$,

where $\langle d\rangle$ is the observed mean-value of the distance, $d$.

Another way is to use the mean-density, $\rho$. The acceleration then becomes

$a_{h}=-\frac{4 \pi \rho G d}{3}$.

Let us now obtain a quantitative measure of inhomogeneity. If we start with the real acceleration, we can simply add and subtract the "homogeneous acceleration", $a_{h}$,

$a=a_{h}+\left(a-a_{h}\right)$,

and extract a factor $v^{2} / d$ from the bracket

$a=a_{h}+\frac{v^{2}}{d}\left(\frac{a d}{v^{2}}-\frac{a_{h} d}{v^{2}}\right)$.

Introducing the Hubble parameter, $H=v / d$, we get $a=a_{h}+d H^{2}\left(\frac{a d}{v^{2}}-\frac{a_{h} d}{v^{2}}\right)$,

where the bracket is dimensionless. As can be seen, this inhomogeneous result completely without dark energy yields the same behavior as the standard (homogeneous) model with a cosmological constant [13],

$a=a_{h}+d H^{2} \Omega_{\Lambda}$.

We denote the correction term for the inhomogeneity by

$Q=\left(\frac{a d}{v^{2}}-\frac{a_{h} d}{v^{2}}\right)$

Let us now consider an inhomogeneous "universe" consisting of two large bodies and an observer, whose mass is negligible, situated between them. The two bodies are taken to have equal masses and therefore half the mass of the universe.

As this is a two-body problem the acceleration in the radial direction is

$a=-\frac{G M}{8 d^{2}}$.

The gravitational potential, $V$, is defined according to

$F=-\nabla_{2 d} V$,

where $F$ is the force acting on the particles in the radial direction,

$F=-\frac{G M^{2}}{16 d^{2}}$.

Solving for $V$ gives

$V=-\frac{G M^{2}}{8 d}$

If the bodies have escape velocity (corresponding to flat space-time), the virial theorem states that

$2 K+V=0$,

where $K$ is the kinetic energy of one of the particles, $K=-M v^{2} / f r 4$. Solving for $v^{2}$ gives

$v^{2}=\frac{G M}{4 d}$.

The total mass of the Universe is $M$ so,

$a_{h}=-\frac{27 G M d}{64\langle d\rangle^{3}}$.

In this case $\langle d\rangle=d$ because both particles are at the same distance from the observer. So the "homogeneous acceleration" becomes

$$
a_{h}=-\frac{27 G M}{64 d^{2}},
$$

which gives the value, 


$$
Q=\left(-\frac{1}{2}+\frac{27}{16}\right)=1.2
$$

Thus the real acceleration will be

$$
a=a_{h}+d H^{2} 1.2
$$

The motivation for this somewhat trivial demonstration is to show that if we observe an inhomogeneous Universe and still "pretend" it is homogeneous, it will yield a correction term for the inhomogeneity that behaves in the same way as a cosmological constant, as can be seen by comparing Eqs. (13) and (24). This points to the possibility to remove the need for a mysterious dark energy - which today has no fundamental, microscopic explanation or justification whatsoever.

An N-body simulation with newtonian gravitation that takes into account the effects of special relativity $\left(p=m_{0} v \rightarrow p=m v\right)$ has been done. The particles have a small gas-like extension, $r$, so if they get really close to each other the gravitational potential will not be infinite. If the particles are separated by a distance larger than $r$ the potential is

$V=-G \frac{m^{2}}{d}$

where $m$ is the mass of each particle, while for $d<r$ it is

$V=-G \frac{3 m^{2}}{2 r}+G \frac{m^{2} d^{2}}{r^{3}}$.

The particles will therefore not collide but simply pass through each other without obtaining an infinite acceleration, just as expected for the "test-particles" of cosmology; galaxies. They are distributed randomly in a sphere so that the large-scale density is almost uniform. The particles begin with escape velocity as initial condition, as this is the Newtonian equivalent to a flat universe in the Einstein setting. The acceleration is calculated and the next distance and velocity for all particles are iterated using a finite difference method. Plotting the distance from the center of the sphere against red-shift, and comparing to a few general relativistic "standard" cosmological models, show some interesting results, Fig. (2). All models give similar behavior up to $z \sim 1$ and the particles in the flat but inhomogeneous model approach the homogeneous open model for higher $z$.

We end with some related comments:

- In a FRW-universe with a cosmological constant it is just a strange and completely unexplained "cosmic coincidence" that $\Omega_{M} \sim \Omega_{\Lambda}$ now $\left(\Omega_{M}>>\Omega_{\Lambda}\right.$ earlier and $\Omega_{M} \ll \Omega_{\Lambda}$ later). However, in our scenario it is an automatic bonus, as an appreciable amount of structure must form before intelligent life can evolve to observe it. It is thus natural that we live in an epoch when the apparent "acceleration" (really due to inhomogeneity) becomes observable.

- SN Ia data probe regions with $z \leq 1.7$. Homogeneity and isotropy is valid only on scales significantly larger (orders of magnitude) than the cosmological "voids" and "filaments" $[11,12]$, i.e., at distances > $120 \mathrm{Mpc}$

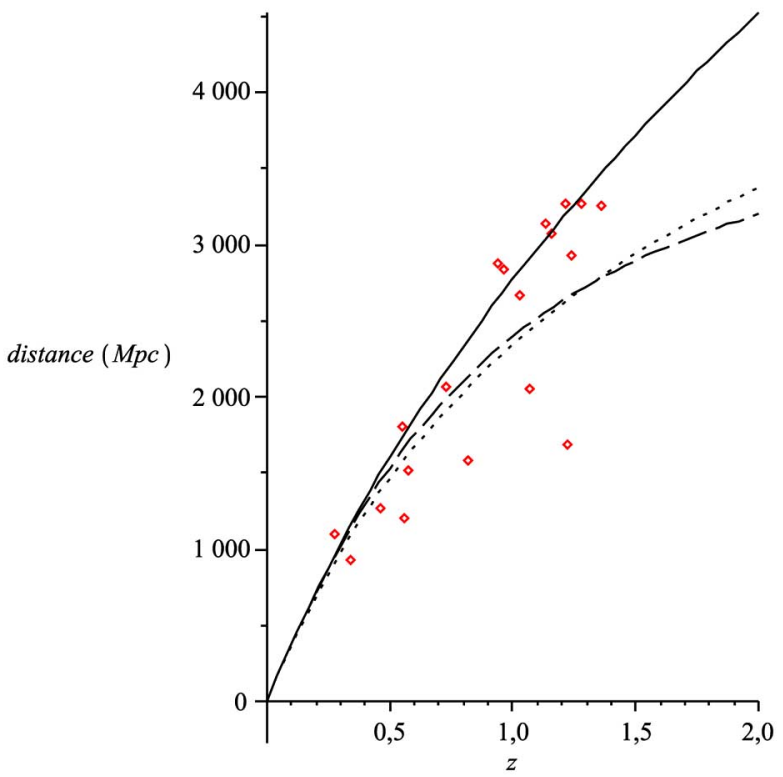

Fig. (2). The prediction of three different homogeneous models are shown together with our simulated inhomogeneous "particles". The solid line is an open FRW-model with $\Omega_{M}=0.2, \Omega_{\Lambda}=0$. The dotted line is a flat FRW-model with $\Omega_{M}=1, \Omega_{\Lambda}=0$. The dashed line is the (flat) newtonian prediction with corrections for special relativity. An inhomogeneous matter distribution may thus be interpreted wrongly as to suggest that $\Omega_{M}$ is lower than it actually is, e.g. $\Omega_{M}=0.2$ if interpreted through a homogeneous and isotropic "standard model" cosmology. The simulated particles correspond to $\Omega=\Omega_{M}=1$. The assumption of homogeneity and isotropy mislead the physical interpretation if the real distribution is inhomogeneous, as is the case for our Universe.

(corresponding roughly to $z \sim 0.03$ ). Neither can one $a$ priori rule out clumping on even grander scales. Hence, the "cosmological principle" of homogeneity and isotropy, which the FRW-solution crucially depends upon, does not apply exactly. Instead full consideration of the inhomogeneities should be taken, at least up to the distance scale where homogeneity and isotropy may be considered a valid approximation.

- The CMBR almost certainly probes the overall geometry/curvature of the universe $(z \sim 1100)$, as little gravitational structure could form/grow before photon decoupling. The statistical weight of the low $z$-range where appreciable structure has formed is negligible compared to the higher $z$-range which thus dominates the integrated effect for the CMBR. For very high redshift the photons accordingly should behave "as expected" in a flat universe. Also, the CMBR is "everywhere" while SN photons travel from a pointlike source to us along a sharp geodesic "ray". This means that, due to the inhomogeneity and anisotropy at small to medium scales, constraints from SN and CMBR may not "carry over" trivially between one another.

In conclusion, we have showed that by regarding the real inhomogeneous matter distribution arising from timedependent gravitational structure formation, it might be possible to avoid the conclusion that the expansion of the 
universe accelerates, as normally drawn from high- $z$ SN Ia data. This would alleviate the need to postulate that the present universe at large is dominated by an exotic "dark energy" with a mysterious negative pressure.

This can also be seen as a plea to start taking seriously the nonlinear character of general relativity, one of the intrinsically most highly nonlinear theories in existence, where even very "small" perturbations could grow exponentially. This, and other phenomena, are known to occur in other (e.g. chaotic) nonlinear systems, whereas overly simplified linearized models, or even the whole perturbative series with infinitely many terms, suppress or even exclude such behavior. Coupled with the vast distances and timescales relevant in cosmology this could make all the difference.

For example, a FRW-model with newtonian perturbations as used in simulations of large-scale structure formation may be overly simplified to capture the true dynamics. We believe that the nonlinear aspects of general relativity so far have been gravely underestimated in cosmology.

Despite high-precision observations advancing cosmology towards becoming a normal "exact science" in the last couple of decades the present physical understanding of the universe as a whole is probably still very crude. We predict that theoretical cosmology fifty years from now will bear little resemblance to the FRW-models almost universally used today.

\section{REFERENCES}

[1] Riess A, Filippenko AV, Challis P, et al. Observational evidence from supernovae for an accelerating universe and a cosmological constant. Astr J 1998; 116: 1009-38

[2] Perlmutter S, Aldering G, Goldhaber G, et al. Measurements of Omega and Lambda from 42 High-Redshift Supernovae. Astrophys J 1999; 517: 565-86.

[3] Ahlenius P, Lundgren A. Distance measurements in inhomogeneous cosmologies. Luleå University of Technology M.Sc.-thesis 2007:121 CIV, ISSN: 1402-1617, 2007.

[4] Kolb EW, Matarrese S, Riotto A. On cosmic acceleration without dark energy. New J Phys 2006; 8: 322-47.

[5] Rasanen S. Accelerated expansion from structure formation. J Cosmol Astopart Phys 2006; 11: 003-43.

[6] Hanany S, Ade P, Balbi A, et al. MAXIMA-1: A measurement of the cosmic microwave background anisotropy on angular scales of 10 arcminutes to 5 degrees. Astrophys J Lett 2000; 545: 5-9.

[7] deBernardis P, Ade PAR, Bock JJ, et al. A flat universe from highresolution maps of the cosmic microwave background radiation. Nature 2000; 404: 955-9.

[8] Kogut A, Spergel DN, Barnes C, et al. Wilkinson Microwave Anisotropy Probe (WMAP) First Year Observations: TE Polarization. Astrophys J Suppl 2003; 148: 161-73.

[9] de Vaucouleurs G. The case for a hierarchical cosmology. Science 1970; 167: 1203-13.

[10] Leibundgut B, Sollerman J. A cosmological surprise: the universe accelerates. Europhys News 2001; 32: 121-5.

[11] Einasto J, Einasto M, Gottlober S, et al. A $120-\mathrm{Mpc}$ periodicity in the three-dimensional distribution of galaxy superclusters. Nature 1997; 385: 139-41

[12] Einasto J. Large scale structure of the universe: current problems. In: Gurzadyan VG, Ruffini R, Eds. The Chaotic Universe, Adv Ser Astrophys Cosmol 10, World Scientific, Singapore 2000; p. 191.

[13] Peebles PJE. Principles of Physical Cosmology. Princeton, University Press: New Jersey 1993.

(C) Hansson and Lindkvist; Licensee Bentham Open.

This is an open access article licensed under the terms of the Creative Commons Attribution Non-Commercial License (http://creativecommons.org/licenses/by-nc/3.0/) which permits unrestricted, non-commercial use, distribution and reproduction in any medium, provided the work is properly cited. 